\title{
Retracted: Information Fields Navigation with Piece-Wise Polynomial Approximation for High-Performance OFDM in WSNs
}

\author{
Mathematical Problems in Engineering \\ Received 26 January 2015; Accepted 26 January 2015 \\ Copyright (C) 2015 Mathematical Problems in Engineering. This is an open access article distributed under the Creative Commons \\ Attribution License, which permits unrestricted use, distribution, and reproduction in any medium, provided the original work is \\ properly cited.
}

The paper titled "Information Fields Navigation with PieceWise Polynomial Approximation for High-Performance OFDM in WSNs" [1], published in Mathematical Problems in Engineering, has been retracted upon the authors' request, as they found that there are some wrong results in their paper.

\section{References}

[1] W. Wei, P. Shen, Y. Zhang, and L. Zhang, "Information fields navigation with piece-wise polynomial approximation for highperformance OFDM in WSNs," Mathematical Problems in Engineering, vol. 2013, Article ID 901509, 9 pages, 2013. 

Approximation for High-Performance OFDM in WSNs

\author{
Wei Wei, ${ }^{1}$ Peiyi Shen, ${ }^{2}$ Ying Zhang, ${ }^{3}$ and Liang Zhang ${ }^{2}$ \\ ${ }^{1}$ School of Computer Science and Engineering, Xian University of Technology, Xi'an 710048, China \\ ${ }^{2}$ National School of Software, Xidian University, Xian 710071, Shaanxi, China \\ ${ }^{3}$ School of Electronic and Information Engineering, Xian Jiaotong University, Xi'an 710049, China
}

Correspondence should be addressed to Wei Wei; weiwei@xaut.edu.cn

Received 23 September 2012; Revised 11 December 2012; Accepted 12 December 2012

Academic Editor: Zheng-Guang Wu

Copyright (C) 2013 Wei Wei et al. This is an open access article distributed under the Creative Commons Attribution License, which permits unrestricted use, distribution, and reproduction in any medium, provided the original work is properly cited.

Since Wireless sensor networks (WSNs) are dramatically being arranged in mission-critical applications, it changes into necessary that we consider application requirements in Internet of Things. We try to use WSNs to assist information query and navigation within a practical parking spaces environment. Integrated with high-performance OFDM by piece-wise polynomial approximation, we present a new method that is based on a diffusion equation and a position equation to accomplish the navigation process conveniently and efficiently. From the point of view of theoretical analysis, our jobs hold the lower constraint condition and several inappropriate navigation can be amended. Information diffusion and potential field are introduced to reach the goal of accurate navigation and gradient descent method is applied in the algorithm. Formula derivations and simulations manifest that the method facilitates the solution of typical sensor network configuration information navigation. Concurrently, we also treat channel estimation and ICI mitigation for very high mobility OFDM systems, and the communication is between a BS and mobile target at a terrible scenario. The scheme proposed here combines the piece-wise polynomial expansion to approximate timevariations of multipath channels. Two near symbols are applied to estimate the first-and second-order parameters. So as to improve the estimation accuracy and mitigate the ICI caused by pilot-aided estimation, the multipath channel parameters were reestimated in timedomain employing the decided OFDM symbol. Simulation results show that this method would improve system performance in a complex environment.

\section{Introduction}

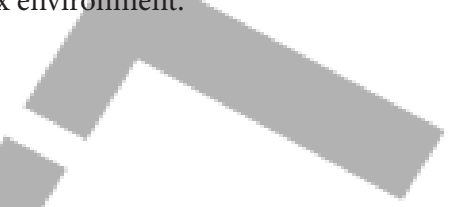

In the past twenty years of active research and field trials, WSNs have started penetrating into many areas of science, engineering, and our daily life. They are also envisioned to be an integral part of cyber-physical systems for example those for alternative energy, transportation, and healthcare. In supporting mission-critical, real-time, closed loop sensing and control, WSNs express a significant departure from traditional WSNs which usually focus on open-loop sensing in WSNs. The impending application requirements in CPS make it necessary to rethink about WSNs design.

Conventional applications [1-8] on distributed data collection systems have already identified the advantages of cheap networked nodes over traditional centralized sensing systems. As a whole, most stochastic individual movements, the simplest of which, the random waypoint mobility model (equivalent to Brownian motion), is adopted to show pure random movements of the entities of a system [4]. That is why efficient and convenient navigation is important to users. In the paper, we work on the information potential of using a sensor network to aid information query and navigation through a dynamic and real-time environment. It includes the navigation of packets (responding client queries from any target node), furthermore the navigation of drivers or automobiles moving in the specified space. For instance users with mobile communicating equipments with surrounding sensors get real-time navigation data. The construction and maintenance costs of these information potentials are varied by the high-frequency queries about the data sources. Most 
of these gradient-based methods $[9,10]$ employ the natural gradients of physical phenomena, due to the spatial distribution of many physical quantities, for instance, temperature measurements for heat, follows a natural diffusion law.

The novelty of our method is to set up a practical and convenient information potential field that facilitates clients to discover the local suboptimal value points as free parking spot. Our means can help users to reach the local area with higher density of information field instead of one extremum value point. The area of higher density of information field cannot attract any fierce conflict since this method guides the car to the higher density of information area that keeps several vacancies for cars. It would avert lower level competition for parking spots. The function of the heat conduction equation is the time as the resource refreshed to meet the physical means in the form of pairs of the information on the degree of each node to be updated. Specially, we simulate an information diffusion process by using Heat Equation with boundary values specified. Effectively the information potential set up a smooth "hill area" (information potential field hill) with several local "summits"; almost all nodes on this area are probably to have several ascending neighbors, and thus greater capacity to reach the various definitions. This trick of smoothing out the discrete hop counts by a heat equation can also be applied in other settings where smooth potential fields of information flow require to be maintained. Eventually, we find that others [11] have also used similar concept motivated by the solution to information navigation in sensor networks [8]. References $[6,12]$ use routing based upon an electrostatic potential field and information field; but in those papers the emphasis is on network capacity but not on eliminating competition crisis and convenient information discovery, which are the topics explored in this paper.

The rest of the paper is organized as follows. In Section 2, we give the fundamental mathematical formulations about information diffusion and heat equation. Then, a surface fitting model based on variation method is introduced and a smoothing large-scale (global aspect) information field is established. In Section 3, fundamental mathematical formulations about Laplace equation are described and a continuous small-scale (local aspect) information field is established. In Sections 4, 5, and 6, we provided the high-performance OFDM evaluation results. Simulation numerical examples are offered. The conclusions are given in the last section.

\section{Smooth Large Scale Information Potentials}

Information resources are altering momentarily from the time users receive the guidance signal to the time drivers access the target node. It is a process that client accesses the target node after he receives the guidance information. Nonetheless, in the whole network, information resources are continuously changing dynamically. Therefore, we can take use of multiresolution gradient to accomplish this navigation process; in other words, we can solve various cases of navigated targets with different levels. First, customers are enquired to arrive the better area with the higher information level, which means to finish the inaccurate navigation or fuzzy guidance. This configuration navigator is a quick and fuzzy process that cannot guarantee the client reach the specific parking lot. But it can assist the customer reach the certain area that holds some extent information field. And then, it could finish the further guidance to get in the assured point. For the undetermined configuration navigator, we require to construct a large scale and smooth information gradient field (global information gradient) in view of the inaccurate gradient descent method.

2.1. Information Diffusion and Heat Equation. The original information gradient field of the entire network is precise in each target node. But it cannot show the information level of local area. For the uncertain configuration navigation, it is necessary for the client to find a field reflecting local information level. For this local information field, the information is not independent among various nodes but also affect each other. Consequently, some extent diffusibility of the information should be considered in the whole network. Each node will extend its own valid information as source node. The information diffusing process is represented by a diffusion equation (that is to say, heat flow equation, a kind of PDE). In this equation, $c$ is Normal number, means the proliferation of the speed. As time goes, the original information fields gradually become a constant field. This result is not useful for resolving the problem. For evolving the information field to a better smooth version that preserved the major features, the diffusing speed should be controlled and then the following diffusion equation (Heat equation) is introduced:

$$
\begin{gathered}
\frac{\partial u}{\partial t}=F(u) \nabla^{2} u \\
u(0, x, y)=u_{0}(x, y),
\end{gathered}
$$

where the function indicates for the diffusion speed of every node and the function expresses the adjacent area of the position $(x, y)$. The central-difference scheme is used to solve (1). A smooth information field that reflects the local information level will be obtained after 2257 times iterations as shown in Figure 1. The relative change is less than $0.01 \%$.

2.2. Potentials Fitting Based on Variation Method. To acquire the large-scale smooth information field, the gradient descent method is used to navigate the car by the information resource. For the above Section 2.1, it is prerequisite for us to snatch the more accurate grid and fitting the information field layer. Thus, it is natural to refer to the energy functional.

Mesh refinement is performed directly. The four vertexes of each grid hold the specific value, and then it can decide a quadrilateral space. On account of the position inside the defined triangle within the quadrilateral space, the information gradient of the new adding vertex can be computed on the basis of triangular coordinates.

Nonetheless, after completing the refinement, the information gradient surface is still a piecewise triangular plane as before so that it cannot adopt the gradient method. Balance between accuracy and smoothness defined the following PDE 

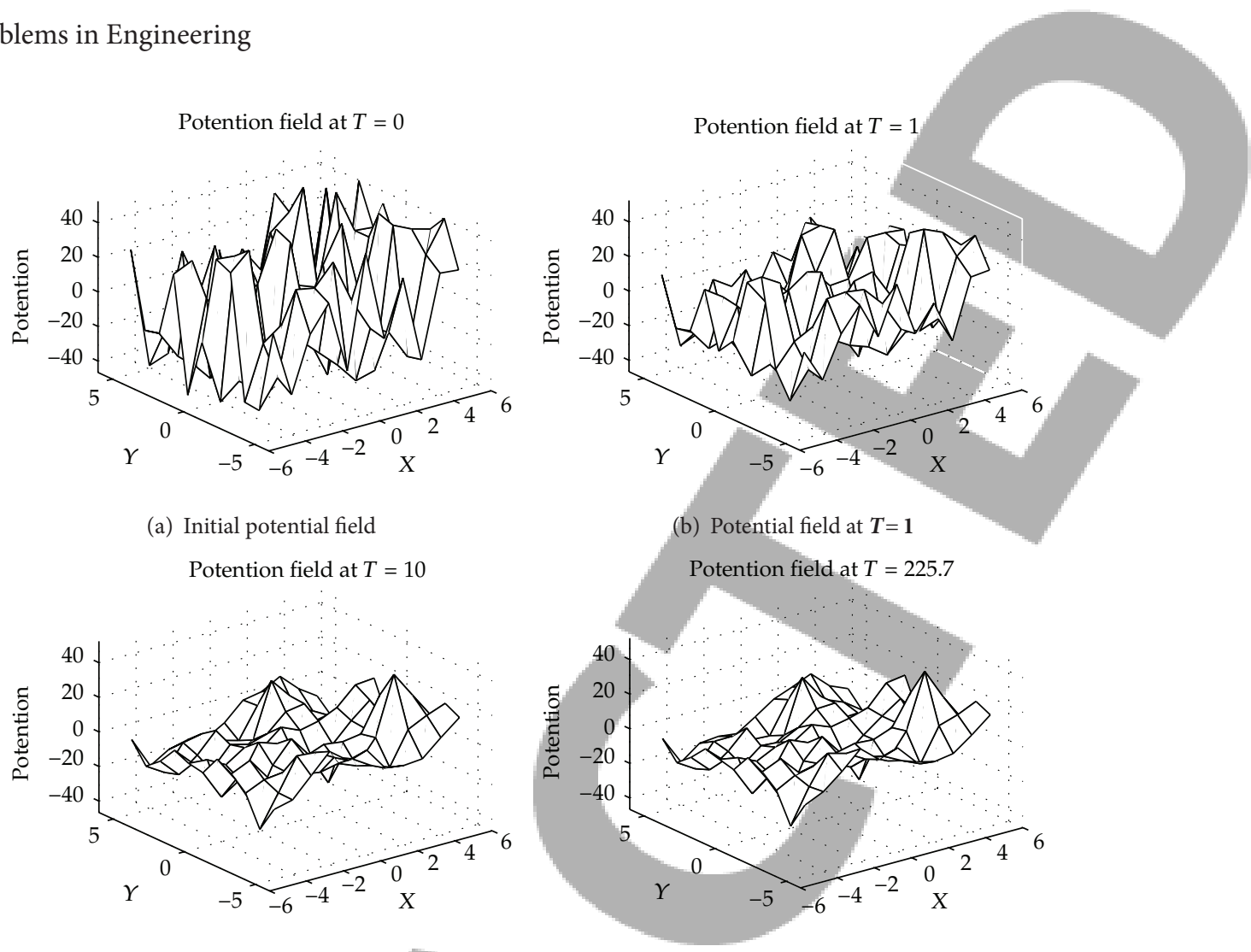

(a) Initial potential field

Potention field at $T=10$

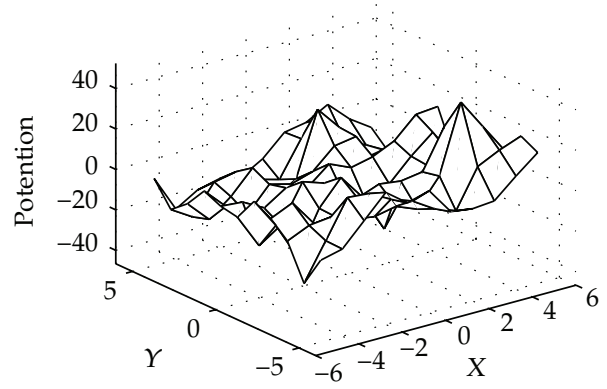

(d) Final potential field

(c) Potential field at $\mathbf{T}=\mathbf{1 0}$

FIGURE 1: Evolution of information field under the controlling of variable coefficients diffusion equation.

to measure the smoothness of the curved surface and measure the deviation between one surface and the initial surface (the piecewise triangular surface)

$$
\varepsilon(v)=\alpha \int_{\Omega}(v-u)^{2} d \Omega+\beta \int_{\Omega}|\nabla v| d \Omega+\gamma \int_{\Omega}|\nabla v|^{2} d \Omega .
$$

In the above functional, $\alpha$ and $\beta$ are normal parameters. To get an optimal surface $v^{*}(x, y)$ (this surface is smooth enough and the deviation between it and $u(x, y)$ is small enough), the following optimal problem can be built up.

It is needed to search $v^{*}(x, y)$ that satisfy $\varepsilon\left(v^{*}\right)=$ $\min \left\{\varepsilon(v) \mid v \in H_{0}^{1}(\Omega)\right\}$ where $H_{0}^{1}(\Omega)$ the Soblev space of is $\Omega$.

Consequently, computing the Euler-Lagrange equation of energy functional (2), and then we introduce the additional part $\partial u / \partial t$ so that the corresponding evolution equation is obtained as follows:

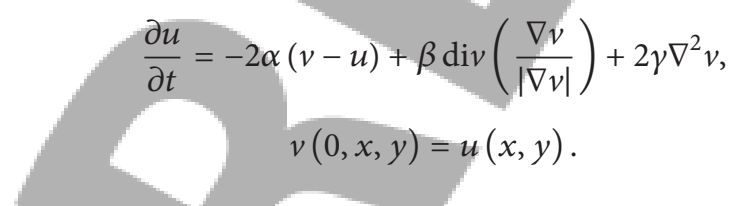

Set $\alpha=1, \beta=1, \gamma=5, \tau=0.01$ and set the relative error limit as $0.01 \%$. The central-difference scheme is used in computation and a smooth large-scale potential field (see also Figure 2) can be obtained after 478 times iterations.
After performing the construction of the large-scale smooth information potential field, we can utilize the gradient descent method to navigate clients.

\section{Small-Scale Information Potentials Based on Laplace Equation}

By the uncertain configuration navigation information of the large-scale information field, drivers reached the higher information field level, and then, customers need to be navigated accurately within the small-scale (local) information field. Definitively, clients could reach a certain node and accomplish the requirement. To fulfill the last configuration navigation object, smooth information is established that it is precise on each local node. The result of La-place equation boundary value problem is beneficial for this goal as follows:

$$
\begin{gathered}
\nabla w^{2}=0, \quad(x, y) \in D, \\
w(0, x, y)=v(x, y), \quad(x, y) \in \partial D .
\end{gathered}
$$

It is affirmed that the value of any internal point can be determined uniquely if the values of the information field function on the boundaries of the nonconvex area are given. What is more, based on the maximum principle, the certain extreme points must lay the boundary. We are enlightened by the previous work ofLin et al. (see in [11]) (They provide a method that set up a smooth information field based 


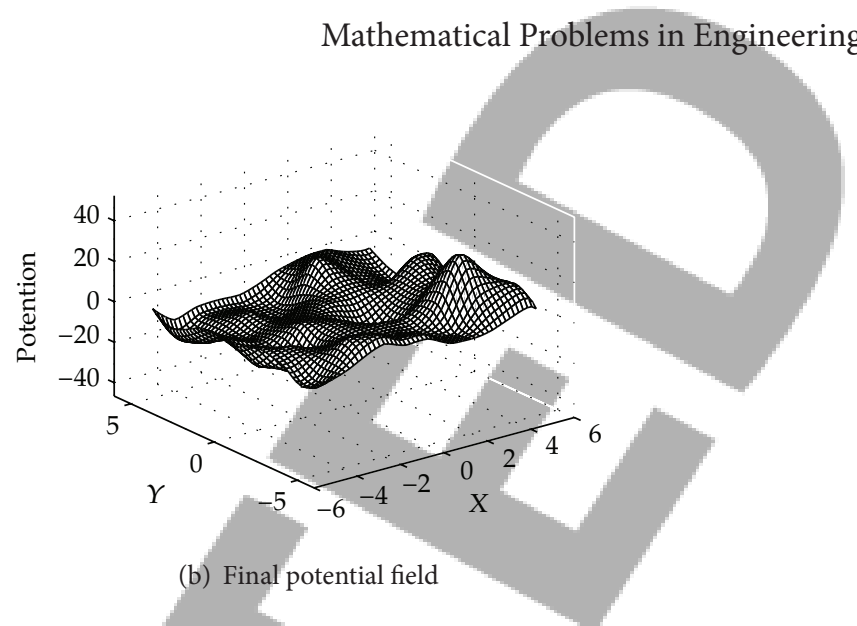

FIGURE 2: Evolution of potential field under the controlling of variable coefficients diffusion equation.

on the limited boundary conditions.) Therefore, a smallscale method is constructed. Figure 3 shows several Laplace problem cases on the nonconvex field.

Consequently, we can establish similar Laplace problem in the current node where lies of the local area so that obtain the small-scale information potential field $w(x, y)$ (in the local node, the value is accurate but not smooth) that is almost smooth everywhere. After computation, then the current node will be refreshed as the local maximum point of the small-scale potential field $w(x, y)$. This node must be one of the nodes of the original discrete information potential field $u_{0}(x, y)$ that meet the requirement of the information level.

\section{Performance Evaluation}

4.1. Implementation. As a result of in the large parking lot real scenarios, it often accompanies with the signal interference and obstacles influence the efficient communication as vehicles are moving quickly. The communication quality is demanded to guarantee for the efficient navigation. We rely on the orthogonal frequency division multiplexing (OFDM) to resolve this problem. (OFDM) modulation avoids intersymbol interference (ISI) by separating a broadband channel into lots of orthogonal narrow-band subchannels [1]. But, for high-mobility applications, the variation of the wireless channel within an OFDM symbol damages the orthogonally and causes intercarrier interference (ICI) [2]. With the velocity increases, if the ICI is not abolished, the system performance will be severely degraded and result in a floor. Accurate channel estimation is necessary to effectively mitigate ICI. Nonetheless, doublyselective channel estimation is a challenge due to the vast number of channel parameters to be estimated. In [3], a fundamental expansion model (BEM) is used to characterize channel timevariations and decrease the number of parameters. Two methods relied on the linear model were proposed in [4] to mitigate ICI in an OFDM system. The correlation of channel response was exploited to improve the estimate accuracy in [5]. Moreover, various schemes have also been presented to treat the ICI cancellation in high-mobility OFDM accordingly [6, 7]. These schemes often require lots of dedicated pilots and implement in frequency domain, with the channel estimation accuracy being affected by ICI, especially in high Doppler spread scenario. In this paper, we are regarded with time-varying channel estimation and ICI mitigation of an OFDM system at very high-speed terminals, for example high-speed railways or MegLev. We are inspired the idea from [4] to obtain the initial polynomial parameters in frequency domain. Then both the first-order and second-order parameters were counted from adjacent symbols and current symbol. To abrogate the ICI caused by frequency-domain estimation and improve the estimation accuracy, decision feedback was performed once to reestimate the channel parameters in time domain. Simulation results show that the proposed scheme improves the mean square error (MSE) of channel estimation the same as the bit error rate (BER) performance. Particularly, the scheme does not need to know the maximum Doppler spread or the statistics of the multipath channel.

4.2. Channel Model. Concernimg a time-varying multipath channel with $L$ taps, the complex baseband representation can be determined by

$$
h(t, \tau)=\sum_{l=0}^{L-1} \gamma_{l}(t) \delta\left(\tau-\tau_{l}\right),
$$

where $\tau_{l}$ is the delay of the $l$ th tap and $\gamma_{l}(t)$ is the corresponding complex amplitude. $\gamma_{l}(t)$ 's are independent for different paths. Assume $\gamma_{l}(t)$ has the same normalized correlation function $r(\Delta t)$ for all $l$, therefore,

$$
r_{\gamma_{l}}(\Delta t)=E\left\{\gamma_{l}(t+\Delta t) \gamma_{l}^{*}(t)\right\}=\xi_{l} r(\Delta t),
$$

where $E\{\cdot\}$ indicates expectation, $\xi_{l}$ is the average power of the $l$ th path. We assume that the average power of the multipath channel is unit $\sum_{l=0}^{L-1} \xi_{l}=1$.

$r(\Delta t)$ is dependent on the vehicle speed or, equivalently, the maximum Doppler frequency. For the classical Jakes' model,

$$
r[m] r\left(m T_{s}\right)=J_{0}\left(2 \pi m f_{d} T_{s}\right),
$$




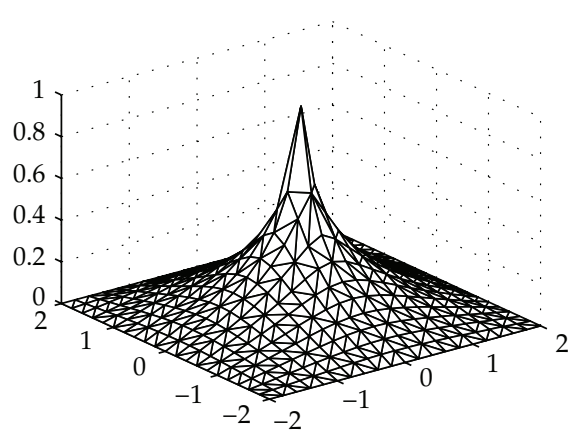

(a)

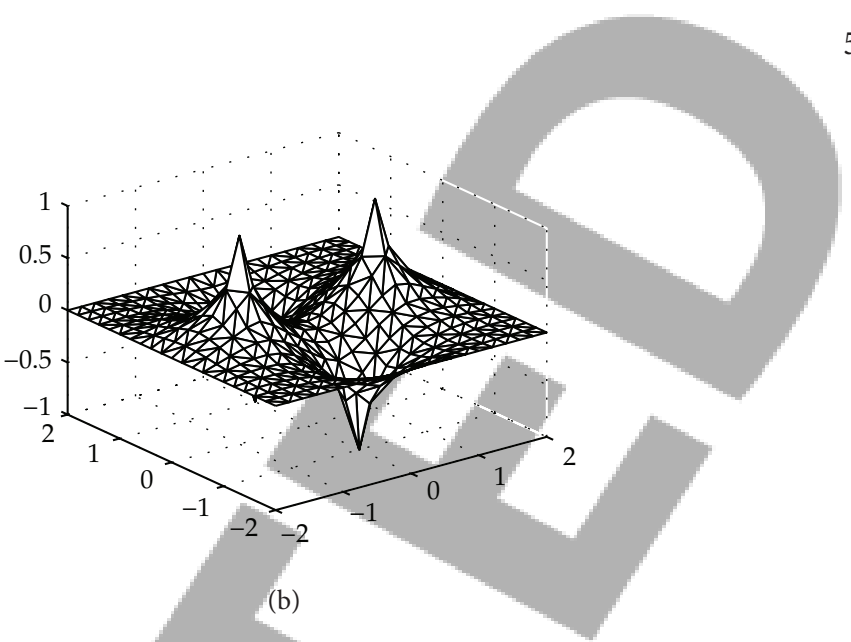

(b)

FIGURE 3: Results of Laplace problems in several nonconvex fields.

where $T_{s}$ denotes sampling interval, $J_{0}(\cdot)$ denotes the zerothorder Bessel function of the first kind. $f_{d}=v f_{c} / c$ is the maximum Doppler frequency with $v$ the speed of the vehicle, $f_{c}$ the carrier frequency, and $c$ the speed of light.

4.3. ICI in Time-Varying Channel. Indicate $X_{k}^{(i)}, 0 \leq k \leq$ $N-1$ as the transmitted signal over the $k$ th subcarrier on the $i$ th OFDM symbol, and then the time-domain transmitted signal can be obtained by

$$
x_{n}^{(i)}=\frac{1}{\sqrt{N}} \sum_{k=0}^{N-1} X_{k}^{(i)} e^{j 2 \pi n k / N}, \quad-N_{g} \leq n \leq N-1,
$$

where $N_{g}$ denotes the length of cyclic prefix (CP). Assume the duration of CP is longer than the maximum delay spread, so that the ISI can be completely avoided. Omit the superscript (i), the time-domain received signal can be obtained as

$$
y_{n}=\sum_{l=0}^{L-1} h_{l, n} x_{(n-l)_{N}}+w_{n}, \quad 0 \leq n \leq N-1,
$$

where $h_{l, n}, 0 \leq l \leq L-1$ denotes the discrete-time channel over the $l$ th path in the $n$th sampling interval, $w_{n}$ indicates the additive white Gaussian noise (AWGN), and $\langle\cdot\rangle_{N}$ is the modulus of $N$.

From (8) and (9), the corresponding frequency-domain received signal over the $m$ th subchannel can be obtained by

$$
\begin{aligned}
Y_{m} & =\frac{1}{\sqrt{N}} \sum_{n=0}^{N-1} y_{n} e^{j 2 \pi m n / N} \\
& =G(m, m) X_{m}+\sum_{\substack{k=0,(15) \\
k \neq m}}^{N-1} G(m, k) X_{k}+W_{m},
\end{aligned}
$$

where $W_{m}$ indicates frequency-domain white noise and $G(m, k)$ denotes channel gain from the $k$ th to the $m$ th subchannel given by

$$
G(m, k)=\sum_{l=0}^{L-1} H_{l}(m, k) e^{-j 2 \pi k l / N}
$$

where $H_{l}(m, k)$ denotes the frequency component of $h_{l, n}, 0 \leq$ $n \leq N-1$, over the normalized frequency $(m-k)$,

$$
H_{l}(m, k)=\frac{1}{N} \sum_{n=0}^{N-1} h_{l, n} e^{-j 2 \pi(m-k) n / N}
$$

In (10), the first item means the desired signal from the $m$ th subchannel and the second item denotes the aggregate ICI from the rest $N-1$ subchannels. Equation (11) indicates that when $k \neq m, G(m, k)=0$ only if $h_{l, n}=h_{l}$. Videlicet, the variation of the wireless channel within an OFDM symbol, causes ICI.

Indicate $\mathbf{Y}=\left[Y_{0} Y_{1} \cdots Y_{N-1}\right]^{T}, \mathbf{X}=\left[X_{0} X_{1} \cdots X_{N-1}\right]^{T}$, and $\mathbf{W}=\left[W_{0} W_{1} \cdots W_{N-1}\right]^{T}$, the frequency-domain received symbol can be denoted by

$$
\mathbf{Y}=\mathbf{G X}+\mathbf{W},
$$

where $\mathbf{G}$ is the $N \times N$ channel frequency-domain response matrix with its element being $G(m, k)$. With a slow timevariant channel, $\mathbf{G}$ can be approximately regarded as diagonal. Nevertheless, as $f_{d}$ increases, the ICI terms on the offdiagonals of $\mathbf{G}$ cannot be ignored, and $\mathbf{G}$ should be treated as banded or even full matrix.

\section{Channel Estimation and ICI Mitigation}

In case of high-mobility condition, the frequency-domain estimate will be influenced by ICI, and then degrade the MSE performance. The correlation-based schemes also faded in fast dispersive channel. We can design a time-domain estimation scheme under the condition that the channel statistics is not known.

5.1. Piece-Wise Polynomial Approximation. In this condition, the time-domain channel responses of each tap are projected over one OFDM symbol duration $T=\left(N+N_{g}\right) T_{s}$ to a set of polynomial basic functions around the center point, that is,

$$
\begin{aligned}
h_{l, n} \approx & \alpha_{0}^{l}+\alpha_{1}^{l} \times\left(n-\frac{N-1}{2}\right) \times T_{s}+\cdots+\alpha_{\mathrm{Q}}^{l} \\
& \times\left(n-\frac{N-1}{2}\right)^{\mathrm{Q}} \times T_{s}^{\mathrm{Q}}, \quad 0 \leq n \leq N-1 .
\end{aligned}
$$


$\alpha_{q}^{l}, 0 \leq q \leq Q, 0 \leq l \leq L-1$ indicates the $q$ th order channel parameter of the $l$ th tap. Thus the channel state information (CSI) can be obtained by estimating $\alpha_{q}^{l}$.

Substitute (15) into (9), we have

$$
y_{n}=\sum_{q=0}^{Q}\left[\sum_{l=0}^{L-1} \alpha_{q}^{l}\left(n-\frac{N-1}{2}\right)^{q} T_{s}^{q} x_{(n-l)_{N}}\right]+w_{n}
$$

Indicate $\mathbf{y}=\left[y_{0} y_{1} \cdots y_{N-1}\right]^{T}, \mathbf{w}=\left[w_{0} w_{1} \cdots w_{N-1}\right]^{T}$, the time-domain received signal can be denoted by

$$
\mathbf{y}=\sum_{q=0}^{Q} \Delta_{q} \mathbf{x} \mathbf{a}_{q}+\mathbf{w}
$$

where $\Delta_{q}$ is a diagonal matrix with diagonal elements $(n-$ $(N-1) / 2)^{q} T_{s}^{q}$, and $\mathbf{a}_{q}=\left[\alpha_{q}^{0} \alpha_{q}^{1} \cdots \alpha_{q}^{L-1}\right]^{T}$ is the stack of $q$ th order parameters for all $l . \mathbf{x}$ is a $N \times L$ transmit matrix stacked by $x_{(n-l)_{N}}$, with the element of $n$th row and $l$ th column indicated by $x[n, l]=x_{(n-l)_{N}}$.

Furthermore, the channel parameters of all the order are stacked into $\mathbf{A}=\left[\mathbf{a}_{0}^{T} \cdots \mathbf{a}_{\mathrm{Q}}^{T}\right]^{T}$, the data matrix as $\mathbf{D}=$ $\left[\Delta_{0} \mathbf{x} \cdots \Delta_{\mathrm{Q}} \mathbf{x}\right]$, and then (16) can be written as

$$
\mathbf{y}=\mathbf{D A}+\mathbf{w}
$$

In frequency domain, substitute (14) into (11), the matrix G can be represented by

$$
\mathbf{G}=\sum_{q=0}^{Q} \mathbf{B}_{q} \mathbf{H}_{q}
$$

where

$$
\begin{gathered}
\mathbf{H}_{q}=\operatorname{diag}\left\{\operatorname{FFT}\left(\left[\mathbf{a}_{\mathrm{q}}^{T} 0 \cdots 0\right]\right)\right\}, \\
\mathbf{B}_{q}=\mathbf{F} \Delta_{q} \mathbf{F}^{\mathrm{H}} .
\end{gathered}
$$

Here, $\operatorname{FFT}(\vec{v})$ represents the FFT of the vector $\vec{v}, \operatorname{diag}(\vec{v})$ is the diagonal matrix spanned by vector $\vec{v}$, and $\mathbf{F}$ denotes the $N$-point unitary DFT matrix with the element of $F[m, n]=$ $e^{-j 2 \pi m n / N} / \sqrt{N}, 0 \leq m, n \leq N-1 . \mathbf{B}_{q}$ is a $N \times N$ circular matrix constructed from sequence $\operatorname{FFT}\left(\operatorname{diag}\left\{\Delta_{q}\right\}\right)$, its elements are denoted by

$$
\mathbf{B}_{q}[m, k]=\frac{T_{s}^{q}}{N} \sum_{n=0}^{N-1}\left(n-\frac{N-1}{2}\right)^{q} e^{-j 2 \pi n(m-k) / N} .
$$

Matrix $\mathbf{B}_{q}$ can be precalculated and stored in the receiver. Since $f_{d}<f_{s}$, where $f_{s}=1 / T_{s}$ denotes the bandwidth of OFDM symbol, it can be suggested that the channel variation is relative smooth over one symbol. Therefore, we only consider the case when $Q=1,2$.

5.2. Pilot-Aided Initial Estimation. In this section, we will discuss how to estimate $\mathbf{H}_{0}, \mathbf{H}_{1}$, and $\mathbf{H}_{2}$ by pilots, respectively.
5.2.1. Estimation of $\boldsymbol{H}_{0}$. Insert $P \geq L$ equally spaced pilots, $d_{p}$, at subcarriers $z_{p}=(p \times N) / P$ for $0 \leq p \leq P-1$. An initial estimate of $\alpha_{0}^{l}$ can then be acquired at pilot tones as follows:

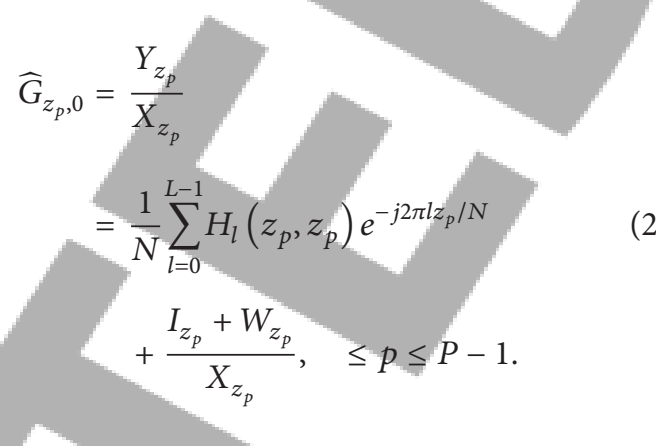

In (22), $I_{z_{p}}$ denotes ICI at $z_{p}$ th subcarrier. Since $H_{l}\left(z_{p}, z_{p}\right)=$ $(1 / N) \sum_{n=0}^{N-1} h_{l, n}$, we can obtain $\widehat{\alpha}_{0}^{1}$ through a $P$-length IFFT of $\left[\widehat{G}_{z_{0}, 0}, \ldots, \widehat{G}_{z_{P-1}, 0}\right]^{T}$,

$$
\widehat{\alpha}_{0}^{l}=\frac{1}{N} \sum_{n=0}^{N-1} h_{l, n}=\frac{1}{P} \sum_{p=0}^{P-1} \widehat{G}_{z_{p}, 0} e^{j 2 \pi p l / P}
$$

Then $\mathbf{H}_{0}$ can be obtained through (8). It should be noted that the pilot-aided parameters estimation is interfered by ICI in (11).

5.2.2. Estimation of $\boldsymbol{H}_{1}$ and $\boldsymbol{H}_{2}$. The adjacent symbols will be employed to estimate the channel parameters when $Q>$ 0 , shown in Figure 4. Generally, three reference points are needed to estimate the second-order polynomial parameter, so the previous and next symbols were used here. Since the polynomial function is expanded at center point, we have $h_{l,(N-1) / 2}^{(i)}=\alpha_{0}^{l(i)}$, where the superscript $(i)$ denotes $i$ th OFDM symbol. For simplicity, the subscript $l$ is omitted owing to independent assumption for each tap.

Assume the fitting curve of current symbol is extended to the midpoint of adjacent symbols, and then $\widehat{\mathbf{H}}_{1}$ and $\widehat{\mathbf{H}}_{2}$ over the $i$ th symbol can be calculated by the following:

$$
\begin{aligned}
& a_{1}^{(i)}= \begin{cases}\frac{a_{0}^{(i+1)}-a_{0}^{(i)}}{T}, & i=1 \\
\frac{a_{0}^{(i+1)}-a_{0}^{(i-1)}}{T}, & 1<i<M-1 \\
\frac{a_{0}^{(i)}-a_{0}^{(i-1)}}{T}, & i=M\end{cases} \\
& a_{2}^{(i)}= \begin{cases}0, & i=1, i=M \\
\frac{a_{0}^{(i+1)}+a_{0}^{(i-1)}-2 a_{0}^{(i)}}{2 T^{2}}, & 1<i<M-1 .\end{cases}
\end{aligned}
$$

5.3. Time-Domain Channel Estimation. In this section we will estimate the polynomial parameters directly in time domain by employing decided symbol, so as to mitigate the effect of ICI. The decided symbol is obtained from initial channel estimation. 
Time-domain channel parameters can be calculated from (17) straightly, the linear MMSE estimate of parameter vector A is

$$
\widehat{\mathbf{A}}_{\text {mmse }}=\left(\mathbf{D}^{H} \mathbf{D}+\sigma^{2} \mathbf{I}\right)^{-1} \mathbf{D}^{H} \mathbf{Y} .
$$

Here, the decided frequency-domain symbol $\widehat{\mathbf{X}}$ is used to construct $\mathbf{D}$. In summary, the procedure of proposed method is listed as follows.

(1) Use(12) to estimate $\widehat{\mathbf{a}}_{0}^{(i)}$ for $1 \leq i \leq M$.

(2) Use (13) and (14) to get $\widehat{\mathbf{a}}_{1}^{(i)}$ and $\widehat{\mathbf{a}}_{2}^{(i)}$ of current symbol.

(3) Substitute $\widehat{\mathbf{a}}_{q}^{(i)}$ into (6) and (8) and get $\widehat{\mathbf{G}}$.

(4) Solve (3) for $\widehat{\mathbf{X}}$.

(5) Construct $\mathbf{D}$ by $\widehat{\mathbf{x}}=\operatorname{IFFT}(\widehat{\mathbf{X}})$, solve (15) for $\widehat{\mathbf{A}}$.

Assume that the multipath channel has $I$ dominant taps, with sampled delays $z_{i}, 0 \leq i \leq I-1$, and then the complexity of time-domain estimation can be reduced to $O\left(Q^{2} I^{2}\right)$, which is trifling for large $N$. Suppose $I_{\text {est }}$ taps are necessity for estimation, $z_{i}$ can be obtained by picking up $I_{\text {est }}$ terms with the largest power from $\widehat{\mathbf{a}}_{0}$, and set the rest to zeroes.

\section{Simulation Results}

In this section, we present simulation results of the proposed scheme using QPSK modulation. The symbol number is $M=10$ and the block size $N=256$. CP length $N_{g}$, pilot number $P$, and the maximum delay $L$ are set to 32 . Symbol duration $T$ is fixed to $0.1 \mathrm{~ms}$ and carrier frequency $f_{c}=3.5 \mathrm{GHz}$, thus the normalized Doppler frequency $f_{d} T=$ 0.162 when the speed is $500 \mathrm{~km} / \mathrm{hr}$. Furthermore, we assume Jakes' Doppler spectrum over multiple paths, the PDP is exponential function with factor 0.05 . In case of missing taps, we estimate $I_{\text {est }}=\mathrm{I}+4$ taps in the scheme.

Figures 5 and 6 show the MSE and BER performance, respectively, when $I=4$ and $I=6$. In the figures, "TD" denotes "time-domain" and "FD" denotes "frequencydomain." $Q=0$ represents traditional FD estimation with time-invariant assumption. As indicated in the figures, both linear $(Q=1)$ and second-order $(Q=2)$ approximation in FD achieve better performance than traditional estimation, despite of high error floors caused by ICI. It can also be watched that TD estimation has further improvement than FD both in MSE and BER performance. When SNR $=20 \mathrm{~dB}$,
TD has a promotion of $8-10 \mathrm{~dB}$ over FD in Figures 5 and 6 , the BER curves of TD are close to that of ideal CSI. Still, there are error floors occurring at very high SNR, which mainly came from the hard decision error. This error floor will be eliminated by iterations.

As the number of dominating taps $I$ increases, the MSE and BER performance degrades. This is reasonable since there are more parameters to be estimated, the performance of FD estimation drops down, which also influences the symbol decision and TD estimation. Figure 5 also indicates that the MSE of $Q=1$ is smaller than that of $Q=2$ in low SNR. This is mainly because second-order fitting have to estimate more parameters, and as estimating error is trifling in high SNR, it will have lower MSE due to the smaller modeling error.

\section{Concluding and Remarks}

In this paper, we proposed a brand new heat diffusion equation to finish the navigation process conveniently and easily. Several partial differential equations are proposed to finish the navigation process conveniently and easily. The partitioned scales are used to reach the goal of the accurate 


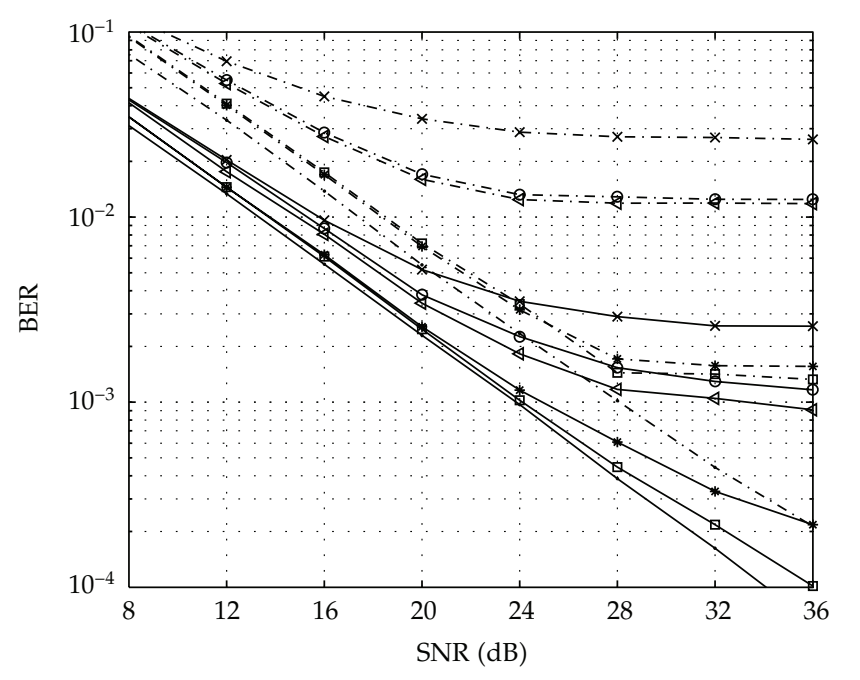

\begin{tabular}{|c|c|c|}
\hline$\times Q=0, \mathrm{FD}$ & 口 & $Q=2, \mathrm{TD}$ \\
\hline ㅇ $Q=1, \mathrm{FD}$ & 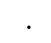 & Perfect CSI \\
\hline$\triangleleft Q=2, \mathrm{FD}$ & 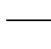 & $I=4$ \\
\hline * $Q=1, \mathrm{TD}$ & $\cdot-\cdot-$ & $I=6$ \\
\hline
\end{tabular}

FIgURE 6: BER Performance versus SNR.

\section{Acknowledgments}

We would like to appreciate the anonymous reviewers for their valuable comments. We also appreciate the Prof. Jing Zhang and Lei Wang for their valuable advices. This work is supported by the financial support from the Natural Science Research Project of Jiangsu Ordinary University (09KJB430008), the Opening Project of State Key Laboratory of High Performance Ceramics and Superfine Microstructure (SKL201111SIC) and Education Reform Project of NJUPT (JG00711JX39). This program is also partially supported by the Open Projects Program of National Laboratory of Pattern Recognition, Natural Science Basic Research Plan in Shaanxi Province of China (Program No. 2010JM8005). And this project is also supported by NSFC Grant (Program No. $61072105,61172018,61007011)$. Our project is also supported by Scientific Research Program Funded by Shaanxi Provincial Education Department (Program No. 11JK0504, 12JK0944, 2010JK723 and No. 12JK0463), by Natural Science Basic Research Plan in Shaanxi Province of China (Program No. 2012JM8047) and by Science and Technology Project of Xian (CX12629) and Xian University of Technology Fund for Distinguished Young Scholars (Program No. 116-211103). navigation. Some theoretic tools such as Heat Diffusion Equation, PDE variational method, and gradient descent methods are adopted in our method. Two smooth potential fields of sensor network are beneficial to satisfy the customers' requirement. Multiscale gradient descent methods, examples, and solid mathematical principles show that the method is accurate and efficient, which enables to solve typical sensor network configuration information navigation. The structure of nonlinear PDE allows more flexibility and adaptability in searching algorithm designs. Compared with the former jobs depending on the discrete information field, our method ensures a local information field large enough to include appropriate multiple targets and the competition conflict can be resolved simultaneously. The information level of each node can be updated with a satisfied physical methodology when resource is dynamically changing. By developing an algebraic structure of heat diffusion equation, we can combine different potentials to enable far greater path diversity and thus provide better performance than it is possible with only onefold discrete field guidance. The simulation results show that although with much relaxed assumptions, our approach achieves comparable performance with significantly reduced competition collision. Simultaneously, we rely on the strengthened novel method to guarantee the communication quality when the cars moving fast based time-domain channel estimation method to mitigate the effect of ICI and improve estimate accuracy. This scheme is applicable to fast dispersive channel and the situation as channel statistics is not determined. Numerical results have simulated that the proposed scheme can increase both MSE and BER performance compared to the frequency-domain channel estimation. We will further explore this direction in the future.

\section{References}

[1] B. Awerbuch and D. Peleg, "Concurrent online tracking of mobile users," in Proceedings of the Conference on Communications Architecture \& Protocols (SIGCOMM '91), pp. 221-233, ACM, New York, NY, USA, 1991.

[2] C. Buragohain, D. Agrawal, and S. Suri, "Distributed navigation algorithms for sensor networks," in Proceedings of the 25th IEEE International Conference on Computer Communications (INFOCOM '06), April 2006.

[3] A. Coté, A. Meyerson, and L. Poplawski, "Randomizedk-server on hierarchical binary trees," in Proceedings of the 40th annual ACM symposium on Theory of Computing (STOC '08), pp. 227234, ACM, New York, NY,USA, 2008.

[4] A. Einstein, Investigations on the Theory of the Brownian Movement, Dover Publications, 1956.

[5] J. Gao, L. Guibas, N. Milosavljevic, and J. Hershberger, "Sparse data aggregation in sensor networks," in Proceedings of the 6th International Symposium on Information Processing in Sensor Networks (IPSN '07), pp. 430-439, April 2007.

[6] J. Gao, L. J. Guibas, J. Hershberger, and L. Zhang, "Fractionally cascaded information in a sensor network," in Proceedings of the 3rd International Symposium on Information Processing in Sensor Networks (IPSN '04), pp. 311-319, April 2004.

[7] C. Intanagonwiwat, R. Govindan, and D. Estrin, "Directed diffusion: a scalable and robust communication paradigm for sensor networks," in Proceedings of the 6th Annual International Conference on Mobile Computing and Networking (MOBICOM '00), pp. 56-67, August 2000.

[8] M. Kalantari and M. Shayman, "Design optimization of multisink sensor networks by analogy to electrostatic theory," in Proceedings of the IEEE Wireless Communications and Networking Conference (WCNC '06), pp. 431-438, April 2006.

[9] J. Liu, F. Zhao, and D. Petrovic, "Information-directed routing in ad hoc sensor networks," IEEE Journal on Selected Areas in Communications, vol. 23, no. 4, pp. 851-860, 2005. 
[10] Z. Tang, R. C. Cannizzaro, G. Leus, and P. Banelli, "Pilotassisted time-varying channel estimation for OFDM systems," IEEE Transactions on Signal Processing, vol. 55, no. 5, pp. 22262238, 2007.

[11] H. Lin, M. Lu, N. Milosavljević, J. Gao, and L. J. Guibas, "Composable information gradients in wireless sensor networks," in Proceedings of the International Conference on Information Processing in Sensor Networks (IPSN '08), pp. 121-132, April 2008.

[12] K. Zhang, Q. Zhang, T. Jiang, and P. Guo, "Demo abstract: a navigation system based on a sensor network without exit and locations," in Proceedings of the 7th ACM Conference on Embedded Networked Sensor Systems (SenSys '09), pp. 311-312, November 2009.
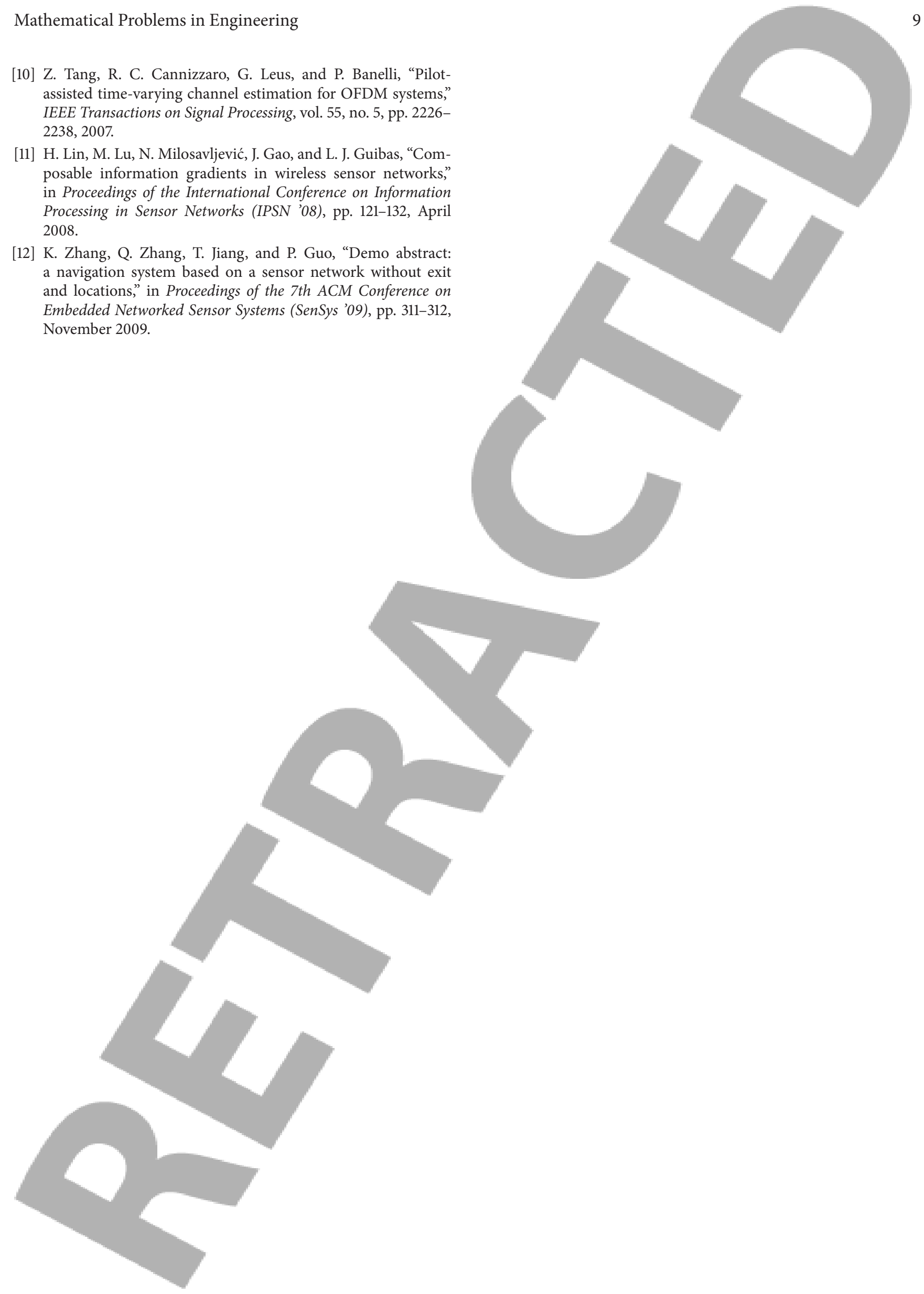